Pathophysiology

of Haemostasis and Thrombosis
Pathophysiol Haemost Thromb 2005;34:255-262

DOI: $10.1159 / 000093104$
Received: June 3, 2005

Accepted: September 2, 2005

\title{
The Extent of Platelet Activation under Shear \\ Depends on Platelet Count: Differential Expression of Anionic Phospholipid and Factor Va
}

\author{
Katherine Schulz-Heik ${ }^{a}$ Jaimohan Ramachandran $^{b}$ Danny Bluestein ${ }^{b}$ \\ Jolyon Jesty ${ }^{\mathrm{a}}$ \\ ${ }^{a}$ Division of Hematology, and ${ }^{\mathrm{b}}$ Department of Biomedical Engineering, Stony Brook University, \\ Stony Brook, N.Y., USA
}

\section{Key Words}

Shear stress - Anionic phospholipid · Factor Va •

Platelet activation

\begin{abstract}
It is widely accepted that shear stress activates platelets. However, this may have two linked but separate causes: a direct effect of shear stress on individual platelets, and secondary inter-platelet activation dependent on the release of agonists caused by shear. Gel-filtered platelets were exposed to intermittent low shear at 20,000 and 200,000 platelets $/ \mu l$ and their activation was measured with a prothrombinase-based assay. At the lower count, activation was slow and essentially linear, but at the higher count, it rose exponentially with time, leading to 3-fold more prothrombinase activity. Inclusion of apyrase and/or prostaglandin $\mathrm{I}_{2}$ slightly reduced activation at high platelet counts, but did not abolish the nonlinear kinetics, and antibodies against von Willebrand factor had no significant effect. The contributions of anionic phospholipid and factor $V a$ to the prothrombinase activity were assessed by measurements in the presence of exogenous factor $\mathrm{Va}$. The results strongly suggest that anionic phospholipid appearance is caused directly by
\end{abstract}

shear exposure, but that factor Va release from the $\alpha$ granules is a secondary event and largely the result of platelet-platelet signaling.

Copyright (C) 2005 S. Karger AG, Basel

\section{Introduction}

Exposure to shear stress activates platelets [1-3]. Normal shear stress in the vasculature is less than $10 \mathrm{dyn} / \mathrm{cm}^{2}$ $(1 \mathrm{~Pa})$ in the venous circulation and increases to about $30 \mathrm{dyn} / \mathrm{cm}^{2}$ in narrower arteries and arterioles. However, it may rise much higher in stenotic arteries, caused for instance by atherosclerosis, well exceeding $100 \mathrm{dyn} / \mathrm{cm}^{2}$. While other aspects of the physiology of atherosclerotic vessels are major contributors, it is widely considered that the exposure of blood to high shear in stenosed coronary and carotid arteries is a leading contributor to thromboembolic disease [4]. This conclusion is indirectly but strongly supported by studies of patients with implanted vascular devices that cause abnormal flow patterns. A particularly clear case is seen with prosthetic heart valves, which cause high shear through the generation of abnormal flow patterns around the valve leaflets and also cause an increased risk of thrombosis $[5,6]$.

\section{KARGER}

Fax +4161306 1234

E-Mail karger@karger.ch

www.karger.com
(C) 2005 S. Karger AG, Basel

$1424-8832 / 05 / 0346-0255 \$ 22.00 / 0$

Accessible online at:

www.karger.com/pht
Jolyon Jesty

Division of Hematology, Health Sciences Center

Stony Brook University

Stony Brook, NY 11794-8151 (USA)

Tel. +1 631444 2059, Fax +1 631444 7530, E-Mail jolyon.jesty@sunysb.edu 
It has generally been assumed that shear-induced activation is caused by shear acting directly on the platelets, but the mechanisms are controversial. In particular, we must discriminate between the initial event of what we call platelet activation and subsequent platelet aggregation. While some investigators consider that the interaction of platelets with von Willebrand factor (vWf) plays a central initiating role in platelet activation under high shear [7], others consider mechanical stress alone to be sufficient, at least in vitro. While shear alone can activate platelets, vWf can act synergistically, providing alternative, or additional, pathways of activation, and it is also involved in platelet aggregation [2,8-11]. There are differences in the literature about the level of shear stress required to activate platelets, in the presence of $\mathrm{vWf}$ or not, although to some extent, this depends on the techniques used to measure activation and their sensitivity.

There are three common methods of assessing platelet activation: (1) measurement of anionic phospholipid exposure on the outer platelet membrane, using flow cytometry with annexin $\mathrm{V}[12,13]$; (2) measurement of an activation-dependent epitope, $\mathrm{P}$-selectin (CD62P), which is released from $\alpha$-granules to the platelet membrane and is measured by flow cytometry with anti-P-selectin antibodies [14], and (3) the prothrombinase method, which in a sense combines these two methods in requiring both anionic phospholipid and a released $\alpha$-granule protein, factor Va, and measures the extent to which platelets support prothrombin activation by factor $\mathrm{Xa}$ [15-18]. Apart from its use as an assay of platelet state, we also note that the prothrombinase method is a direct measure of a major functional property of activated platelets. Additionally, unlike most cytometry-based methods, the prothrombinase assay measures the total activity of a preparation under shear, including the contribution of any plateletderived microparticles or lysed platelets. Under shear conditions similar to those reported here, we have estimated the microparticle contribution at approximately $11 \%$ of the total activity over a similar time course [19].

Although the cytometry and prothrombinase methods differ, a fairly straightforward dose-response relation exists, and one may consider the shear dose as being closely related to the integral of shear stress with respect to time $[20,21]$. For example, the conditions we use in this report - an intermittent (25\%) wall sheer stress of $9 \mathrm{dyn} / \mathrm{cm}^{2}$ over $40 \mathrm{~min}$, giving an integral of approximately $90 \mathrm{dyn}$. $\mathrm{min} / \mathrm{cm}^{2}$ - may be considered as roughly equivalent to exposure to a shear stress of $90 \mathrm{dyn} / \mathrm{cm}^{2}$ for $1 \mathrm{~min}$.

Two main methods exist to expose platelets to shear: cone-plate viscometers and flow through a capillary. We have used the latter in several studies and demonstrated that platelet activation in a capillary flow loop is dependent, as required, on flow rate and viscosity, both of which contribute directly to shear stress and time of exposure to shear. In the absence of any shear stress, rates of platelet activation remain minimal. The response to changes in viscosity (shear stress is proportional to viscosity) at constant flow rate conclusively shows that activation is not an artefact of the flow-loop apparatus [21]. This study also provided the first evidence for nonlinear activation kinetics at higher rates of platelet activation.

In addition to shear stress directly causing platelet activation, secondary processes may be expected to occur that depend on the interaction of activated platelets, either directly (as in the formation of aggregates) or indirectly, by the release of agonists. Such platelet-platelet signaling will lead to further amplification of the initial response in a manner analogous to the second wave of platelet aggregation. In previous studies by us and others, platelets have been exposed to shear over a wide range of concentrations, from 5,000 to 300,000 platelets $/ \mu 1$, and the resulting extents of platelet activation vary significantly. For our part, we noted in earlier studies that at a count of 100,000 platelets/ $\mu 1$, the time course of platelet activation was commonly concave, the rate of platelet activation increasing with the time of exposure. We also noted that nonlinearity was more common under conditions of substantial activation, e.g., at higher shear stress or long times of exposure [21]. We considered that under these conditions, activation might represent the sum of (1) initial activation caused directly by shear, leading to (2) the release of platelet agonists, such as thromboxane $\mathrm{A}_{2}$ and ADP, and further, secondary activation. We reasoned that if such crosstalk depends on the platelet release of solution-phase agonists, then the released agonist concentration, and hence the extent of secondary platelet activation, should depend on the platelet count. The importance of platelet count in overall shear-induced platelet activation is relevant not only to experimental studies of platelet activation, but also to platelet function at normal counts in vivo.

\section{Materials and Methods}

Platelets

Informed consent, approved by the Stony Brook University IRB, was obtained from healthy adult volunteers of either sex who had not taken aspirin or ibuprofen for 2 weeks. Thirty milliliters of blood were drawn by venipuncture into $0.3 \mathrm{ml} 40 \%$ trisodium citrate. Gel-filtered platelets were prepared as previously described [22]. 


\section{Platelet Activation State}

Platelet activation state (PAS) was measured by a chemically modified prothrombinase method that measures the rate of thrombin generation from acetylated prothrombin over $10 \mathrm{~min}$ in response to factor $\mathrm{Xa}(100 \mathrm{pmol} / \mathrm{l})$ at a platelet count of $5,000 / \mu \mathrm{l}$. The acetylation of prothrombin blocks any feedback action of the generated thrombin on the platelets or factor Va during the assay, ensuring linear kinetics during the assay and quantitative measurement of activation state [21,22]. From all experiments, PAS values were normalized to the average activity of fully activated platelets measured individually after each experiment, obtained by sonication (10 W for $10 \mathrm{~s}$, Branson Sonifier 150 with microprobe). PAS values are expressed as percent maximal prothrombinase activity. Under these particular conditions of reactant concentrations and platelet count, $100 \%$ activated (sonicated) platelets generate thrombin at a rate of $4-7 \mathrm{nmol} / \mathrm{l} \cdot \mathrm{min}$.

\section{Circulation Experiments}

Circulation experiments were done at $37^{\circ} \mathrm{C}$ in a capillary flow loop [21], producing an intermittent wall shear stress of $9 \mathrm{dyn} / \mathrm{cm}^{2}$. With a 1-meter capillary in the loop, the platelets are exposed to this shear stress for approximately $25 \%$ of the total circulation time. For each loop circuit, the exposure is approximately $25 \mathrm{~s}$ shear exposure (in the capillary) followed by $75 \mathrm{~s}$ minimal shear $(\leq 0.5$ $\mathrm{dyn} / \mathrm{cm}^{2}$, in the pump circuit). Timed samples were removed for PAS assay to determine the time course of platelet activation. It should be noted that while the platelets were exposed to shear stresses at differing platelet counts (20,000 and 200,000/ $\mu 1)$, all assays of activation state were done at the same count $(5,000 / \mu 1)$.

\section{Inhibitor and Antibody Treatment}

In experiments involving inhibitors, prostaglandin $\mathrm{I}_{2}\left(\mathrm{PGI}_{2}\right.$; Sigma-Aldrich) was used at a final concentration of $1 \mu \mathrm{mol} / \mathrm{l}$, and apyrase (grade VII; Sigma-Aldrich) at a final concentration of $1 \mathrm{unit} / \mathrm{ml}$. Additionally, apyrase and $\mathrm{PGI}_{2}$ were added in combination at the same final concentrations. Anti-vWf (murine monoclonal; Calbiochem-Behring) was used at a final concentration of $10 \mu \mathrm{g} / \mathrm{ml}$. These agents were added to the platelets $15 \mathrm{~min}$ before the start of shear-loop circulation.

\section{Factor Va Contribution}

In experiments in which the contribution of platelet-derived factor Va was tested, samples of platelets from circulation experiments were assessed by prothrombinase assays under different conditions: one assay without additional factor Va (the normal assay) and one containing a large excess $(2 \mu \mathrm{g} / \mathrm{ml})$ of exogenous bovine factor Va [23].

\section{Platelet Variability and Statistics}

Pairing. Individual gel-filtered platelet preparations vary widely in both their base activation state and their sensitivity to activation. All daily experiments were designed as pair comparisons (e.g., high platelet count vs. low, or with vs. without inhibitors), so that daily comparisons were done on the same platelet preparation. These pairwise data, obtained from single platelet preparations, were used to calculate the ratios of activation rates between the matched pairs. The daily paired ratios were then collected to produce a mean pair ratio over that entire data set.

Net Platelet Activation Rates. Time courses of platelet activation during shear exposure in the circulation loop, whether linear with time or not, were first fitted by linear regression, to give a net measure of platelet activation rate over the 39-min course of each circulation experiment. This permitted quantitative comparison of platelet activation at the two platelet counts, regardless of whether the kinetics were linear or not. For the key observation in this paper - the dependence of net activation on platelet count (fig. 1) - differences in activation rates were analyzed by Student's $t$ test to determine whether the ratio (of the rates at the two counts) was significantly different from 1.0, i.e. the null condition of there being no difference. In this form of Student's test, $t=(\bar{x}-\mu) /(s / n)$, where $\bar{x}$ is the mean observed ratio, $s$ its standard deviation, $n$ the degrees of freedom, and $\mu$ is the null hypothesis value against which we test, in this case, 1 . In contrast with Student's ordinary $t$ test, where all data for one condition are lumped and tested against all data for another, this analysis method retains the important withindays pairing information.

Curvature. Using linear regression to measure net activation rates conceals any and all nonlinearity. In order to determine the extent of upward curvature of the generation of platelet activity, time courses of PAS versus time were also fitted to a quadratic equation, $P A S=A t^{2}+B t+C$, and the square coefficient $A$ was used as an index of curvature. This is the rate of increase in platelet activation rate. To determine whether activation was significantly nonlinear, the means ( $\pm \mathrm{SD}$ ) of $A$ were tested against the null hypothesis of linear activation, i.e. $A=0$, using the same form of Student's $t$ test (above).

\section{Results}

\section{Platelet Count}

Platelet activation under shear stress was compared at counts of 20,000 and 200,000 platelets/ $\mu 1,32$ determinations being made in each case (fig. 1). By linear regression, the mean net platelet activation rate at 20,000 platelets $/ \mu 1$ was $0.040 \pm 0.005(\mathrm{SEM}) \% / \mathrm{min}$, whereas at 200,000 platelets $/ \mu 1$, it increased nearly 3 -fold to $0.128 \pm 0.017$ $\% /$ min. By pair analysis (see 'Materials and Methods'), the difference was highly significant $\left(\mathrm{p}<10^{-4}\right)$, and it is clear that the overall extent of activation is greatly affected by the platelet count.

It can be seen in figure 1 that, while the initial rates of activation at both counts are fairly similar over the first 5-8 min, activation at the higher platelet count becomes increasingly concave. To quantify this, time-course data were fitted to a quadratic, and the square coefficient of the quadratic fit $(A)$ was used as a measure of curvature. At a platelet count of $20,000 / \mu 1, A=4.23 \pm 1.4 \times$ $10^{-6} \% / \mathrm{min}^{2}$. Slight but definite curvature was evident, as shown by testing this value against the null value of $A=$ 0 , which represents a straight line [p(linearity) $<0.005$ ]. However, at a count of 200,000 platelets/ $\mu 1$, curvature increased more than 6-fold, giving a value of $A=28.3 \pm$ $5.6 \times 10^{-6} \% / \mathrm{min}^{2}\left[\mathrm{p}\left(\right.\right.$ linearity) $\left.<10^{-4}\right]$. (While shear ex- 
Fig. 1. The effect of platelet count on shearinduced activation. Gel-filtered platelets were subjected to an intermittent shear stress of $9 \mathrm{dyn} / \mathrm{cm}^{2}$ in a capillary loop (being under shear for approximately $25 \%$ of the experimental time course), as described in 'Materials and Methods'. Samples were removed at intervals and assayed for PAS, the PAS values being referenced to fully activated platelets obtained by sonication (see 'Materials and Methods'). Platelets were subjected to shear at counts of 200,000 platelets/ $\mu \mathrm{l}(\odot)$ and 20,000 platelets/ $\mu \mathrm{l}(\bullet)$. Thirty-two experimental runs were done for each condition. Measurements of PAS were done at the same platelet count throughout (see 'Materials and Methods'). Lines are mean quadratic fits.

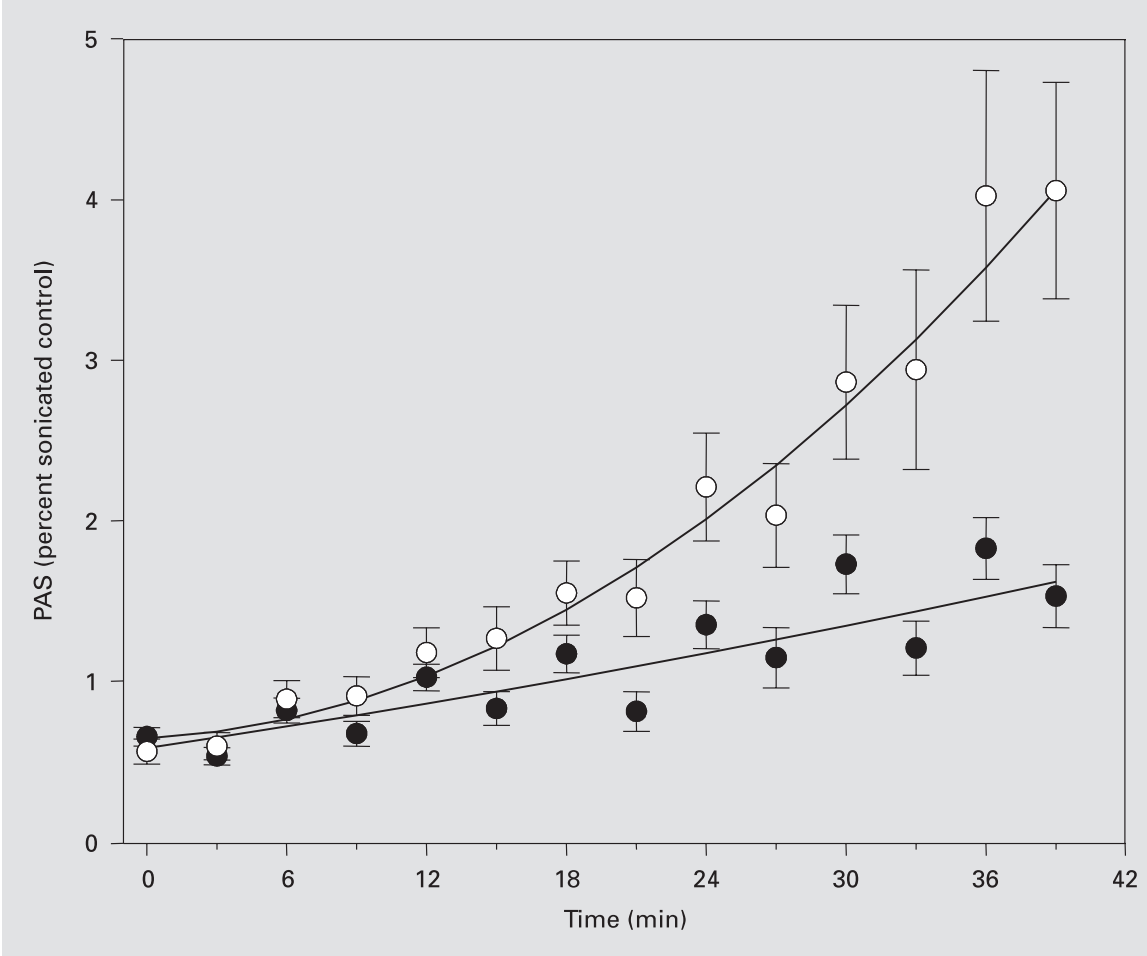

posures were done at different platelet counts, we reiterate that all assays of platelet activation state are done at the same count and are thus independent of it, as shown in 'Materials and Methods'.)

The greatly increased extent of platelet activation and the nonlinearity at the higher platelet count strongly support the idea that shear initiates a platelet-platelet feedback that operates chiefly at higher counts. Positive feedbacks typically produce exponential increases in a rate of reaction, or, in this case, of platelet activation [24]. Although the data could have been fitted to an exponential, $P A S_{\mathrm{t}}=P A S_{0} \exp (k t)$, the quadratic was chosen because the determination of $A$, unlike $k$, is unaffected by variations in the initial activation state, and it also gives a direct measure of the rate of increase in platelet activation rate over the course of an experiment.

\section{Inhibition of Feedback}

As an initial investigation of the mechanism of the apparent feedback, inhibitors of two platelet-released agonists were added to platelets at high counts under shear. ADP, an agonist that is released from platelets on activation, is degraded by apyrase, and the action of thromboxane $A_{2}$, a feedback agonist that is released from platelet membranes, is inhibited by $\mathrm{PGI}_{2}$. When apyrase was add- ed alone ( $1 \mathrm{unit} / \mathrm{ml})$, net platelet activation was essentially the same as in controls at 200,000 platelets/ $\mu$ l (data not shown: $n=5+5 ; \mathrm{p}>0.5$ ). Likewise, when $\mathrm{PGI}_{2}$ was added alone $(1 \mu \mathrm{mol} / \mathrm{l})$, platelet activation rates were the same (data not shown: $n=5+5 ; \mathrm{p}>0.5$ ). When put together (fig. 2a), the two inhibitors produced a small reduction in both net activation rate (by linear regression) and in curvature (by quadratic fit); however, the difference was not significant either $(n=6+6 ; 0.2<\mathrm{p}<0.3)$.

We also investigated a third inhibitor, aspirin, both alone $(n=5 \times 5)$ and in triple combination with apyrase and $\mathrm{PGI}_{2}(n=5 \times 5)$. However, for reasons unknown, pretreatment of platelets with $10 \mu \mathrm{mol} / \mathrm{l}$ aspirin in any combination markedly increased the variability in platelet activation rates in the flow system, and useful statistical analysis was impossible in either case.

\section{Role of $v W f$}

The interior surface of the flow loop is approximately $35 \mathrm{~cm}^{2}$, and gel-filtered platelets contain small amounts of releasable vWf. To determine whether vWf-dependent platelet activation might be occurring, we compared activation in the presence and absence of anti-vWf antibody (fig. 2b). The antibody (mouse monoclonal, $10 \mu \mathrm{g} / \mathrm{ml}$ ) produced a small increase in platelet activation rate $(n=$ 
Fig. 2. The effect of inhibitors of plateletplatelet feedback at high counts. Platelets, $200,000 / \mu 1$, were subjected to intermittent shear stress, as in figure 1: in the presence (•) and absence (O) of $1 \mu M \mathrm{PGI}_{2}$ plus 1 unit/ml apyrase (a), and in the presence of $10 \mu \mathrm{g} / \mathrm{ml}$ monoclonal anti-vWf $(\bullet)$ or $10 \mu \mathrm{g} / \mathrm{ml}$ murine nonimmune $\operatorname{IgG}(\bigcirc)(\mathbf{b})$. a Data show the mean results ( \pm SEM) for 6 control experiments and 6 experiments containing inhibitor. b Results shown are the mean ( \pm SEM) of 4 control experiments and 5 experiments containing antibody. Lines are mean quadratic fits.

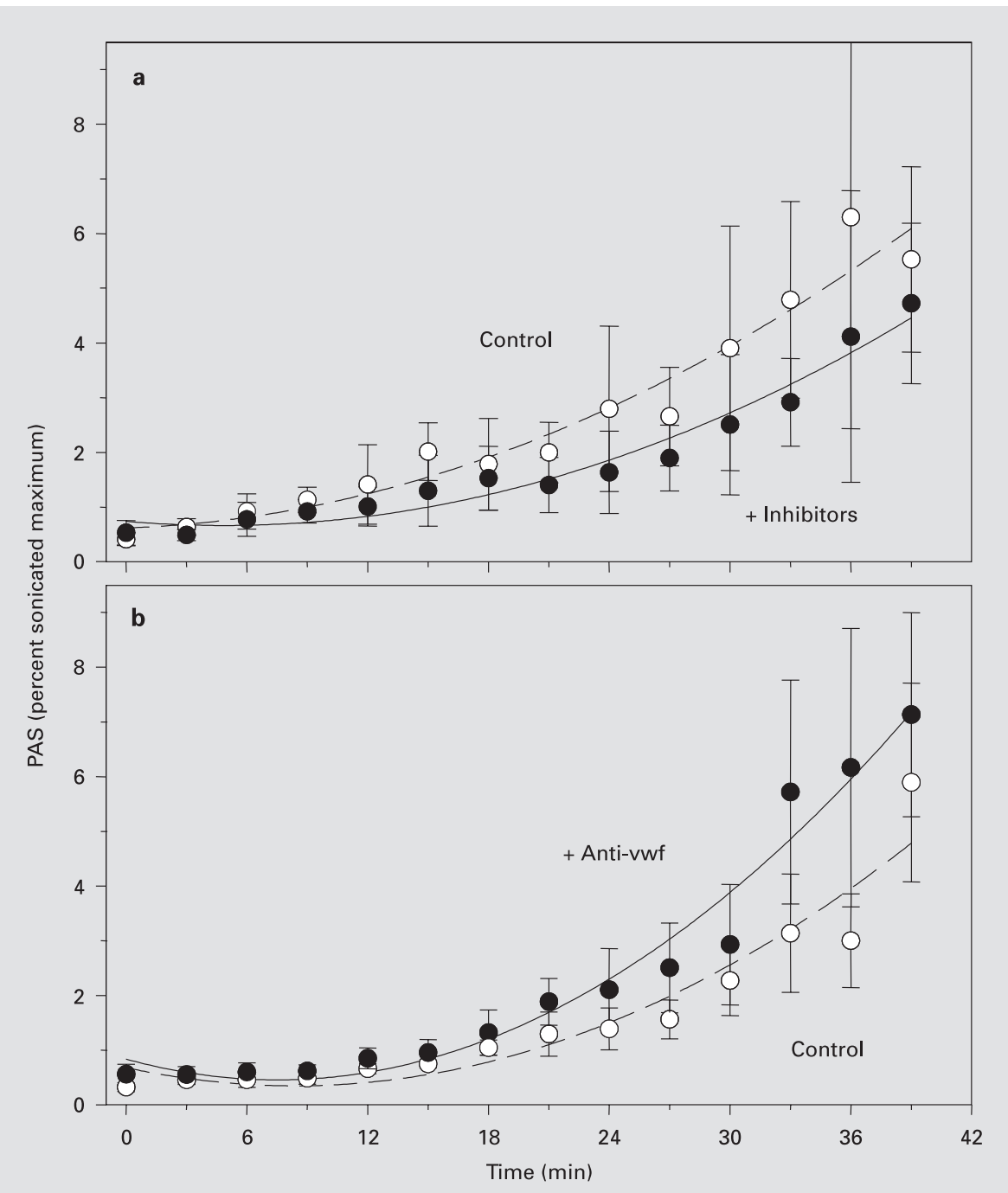

5 antibodies vs. 4 controls), but the difference was not statistically significant $(\mathrm{p}>0.5)$. We conclude that any role of vWf-dependent adhesion and activation in platelet activation under these conditions is at most minor.

\section{Anionic Phospholipid and Factor Va}

Knowing that activated platelets provide factor $\mathrm{Va}$ and anionic phospholipid on their surface, we investigated their relative contribution. Although measurement of annexin $\mathrm{V}$ binding could produce a direct measure of anionic phospholipid exposure, extended real-time access to a flow cytometer would be required, rendering it impractical for our studies. Additionally, we note the wider sensitivity range of the prothrombinase assay, particularly at low levels of platelet activation [21]. Similarly, direct measurement of $\alpha$-granule release by cytometric measurement of P-selectin expression was not feasible. Therefore, we devised a prothrombinase-based alternative to discriminate between phospholipid- and factor Va-dependent activity. We reasoned that in the presence of excess exogenous factor $\mathrm{Va}$, the contribution of platelet-derived factor Va to prothrombinase activity would be minimized, and the measured activation state would mainly reflect the contribution of anionic phospholipid. In other words, if $\alpha$-granule-released factor Va were the limiting source of the concave kinetics of platelet activation observed at higher counts in the ordinary prothrombinase assay, we expected that exogenous factor Va addition would nullify the curvature. This turned out to be the case. 
Fig. 3. Factor $\mathrm{Va}(\mathrm{FVa})$ expression is the primary result of platelet-platelet feedback. Platelets, 200,000/ $\mu 1$, were subjected to intermittent shear stress, as in figure 1 , but were assayed by different methods. a Mean results ( \pm SEM) of 4 experiments that used the standard PAS assay, which measures the platelet contribution of both anionic phospholipid and factor Va. b Results $( \pm$ SEM) of 4 experiments in which prothrombinase activity was measured in the presence of exogenous $2 \mu \mathrm{g} / \mathrm{ml}$ bovine factor Va, isolating the shear-induced contribution of platelet anionic phospholipid. Lines are mean quadratic fits.

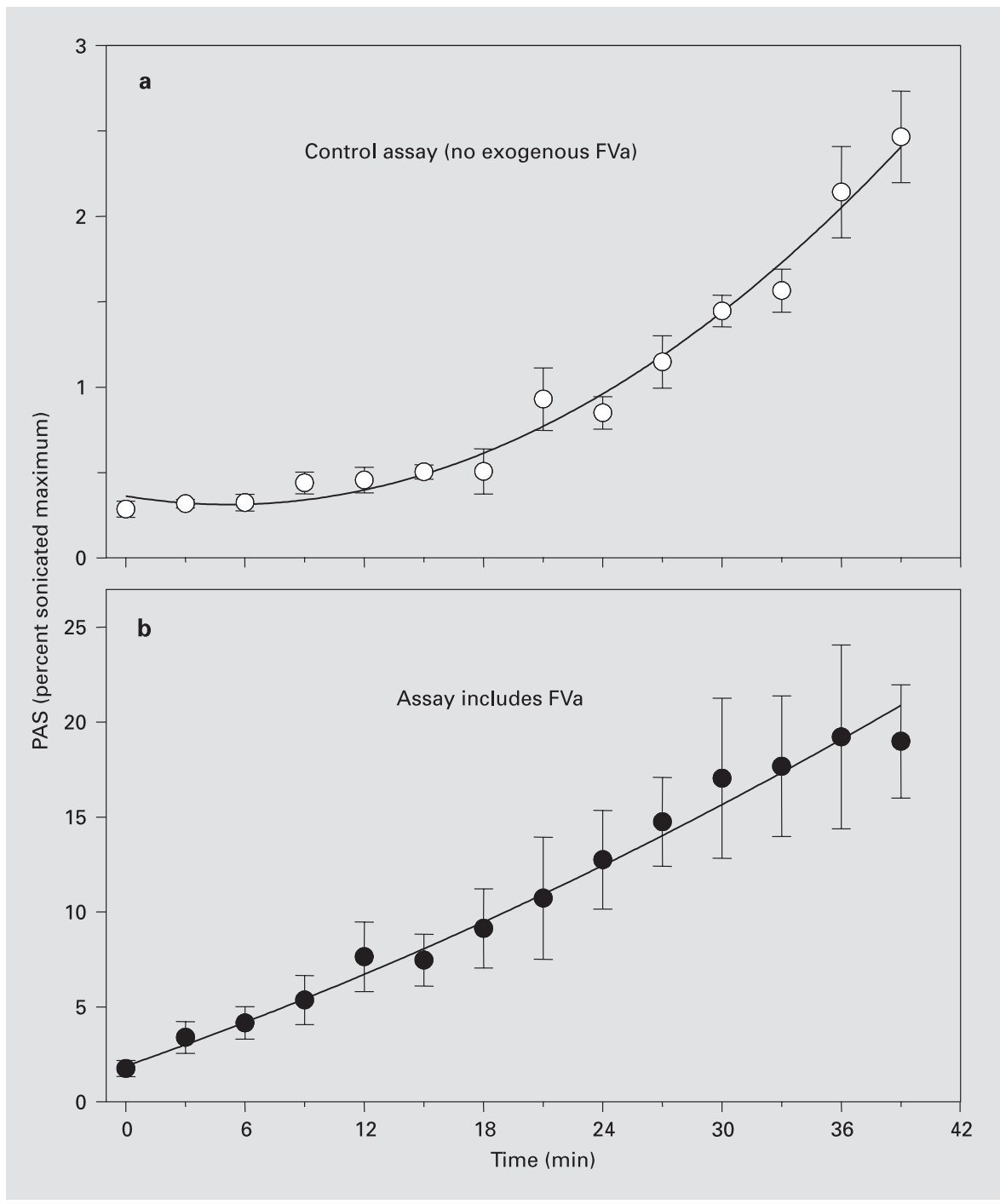

Figure 3 compares platelet activity measured in the normal assay (fig. 3a), where platelets are the sole source of factor $\mathrm{Va}$, and in the modified assay, where excess factor $\mathrm{Va}$ is provided (fig. 3b). In the modified assay, the presence of a high concentration of factor Va greatly increases the rate of prothrombin activation; thus, the actual activation rates and curvature measurements \pm factor Va cannot be directly compared: it is the linearity, or nonlinearity, that is critical. Statistical analysis was restricted to the question of whether activation, as measured in the two assays, was nonlinear. This was done by determining the mean curvature (the square term in a quadratic fit, $A$ ) and testing it against the null hypothesis of linearity $(A=0)$. In the normal assay, in which platelets are the only source of factor $\mathrm{Va}$, and in which factor $\mathrm{Va}$ is seriously limiting, activation is nonlinear, as before $[n=4$; $p$ (linearity) $=0.003$ ] (fig. 3a). In contrast, when excess factor $\mathrm{Va}$ is provided, and when, as we assume, it is mainly the platelet contribution of anionic phospholipid that is being measured, activation is linear $[n=4$; $\mathrm{p}($ linearity $)=0.5$ ] (fig. 3b). This result strongly supports the proposal that factor Va exposure is the major product of platelet-platelet feedback action.

From these results, we propose the following hypotheses: (1) shear stress causes platelet membrane scrambling and anionic phospholipid exposure, and this occurs approximately linearly with time, i.e. in proportion to the time-integrated exposure to shear, and (2) shear-induced activation initiates platelet-platelet signaling, and it is this - and not shear directly - that is responsible for the 
subsequent release of factor $\mathrm{Va}$ from the $\alpha$-granules to the platelet surface. It is this feedback loop that produces nonlinear kinetics.

\section{Discussion}

The results show that the extent of platelet activation upon exposure to shear stress, as measured by the platelet contribution in a prothrombinase assay, depends substantially on the platelet count. This makes clear the importance of particular assay methods in platelet activation studies, and especially, whether an assay measures anionic phospholipid or the release of $\alpha$-granule contents to the membrane, or both. The study does not address the possible role or extent of platelet recovery in the circulation loop. In 'Materials and Methods', we note the intermittent nature of exposure to shear, and it is certainly feasible - particularly at these relatively low shear stresses - that some recovery (i.e. partial restoration of normal membrane phospholipid distribution) occurs after each passage through the capillary section. Nonetheless, activation is shear-dependent [21].

In the flow loop, there are three key parameters that are a direct function of platelet count: the frequency of platelet-platelet collision, the frequency of platelet-wall collision, and the concentration of any soluble agonists that might be released into the medium from shear-activated platelets. We have not addressed the first, and noted that because fibrinogen is absent, these platelets do no aggregate. We have addressed the second briefly, and shown that vWf-dependent effects are at most minimal, suggesting that adhesion is not a primary instigator in this model system. We have investigated the last by adding two inhibitors of important platelet-released agonists apyrase and $\mathrm{GPI}_{2}$ (targeting ADP and thromboxane $\mathrm{A}_{2}$, respectively) - at high platelet counts. Separately, neither had a significant effect. When combined, they did reduce overall activation a little, but platelet activation was not reduced to the level observed at lower platelet counts. We conclude from these results that the release of soluble platelet agonists contributes to, but may not be the sole cause of, increased platelet activation of platelets exposed to shear at high counts.

When the platelet cytoplasmic $\mathrm{Ca}^{2+}$ concentration rises on activation, anionic phospholipid is transferred early to the surface of the membrane bilayer through the action of a $\mathrm{Ca}^{2+}$-dependent scramblase [25]; separately, the platelets release the contents of their $\alpha$-granules, including the release of factor $\mathrm{Va}$ to the membrane in a form that is already substantially active $[15,17,18]$. These are very different processes, and we considered it possible that one or the other might be primary in the expression of prothrombinase activity as measured in the PAS assay. The separation of anionic phospholipid expression from $\alpha$-granule release was also raised in a previous report of the shear response of platelets deficient in IQGAP-1 [26]. It is clear from the actual rates of prothrombin activation shown in figure 3 that factor $\mathrm{Va}$ is severely limiting in shear-activated platelets, and this suggests that the major direct thrombogenic effect of shear exposure is anionic phospholipid exposure and not factor Va release. We propose that factor Va exposure depends largely on plateletplatelet crosstalk, leading to the release reaction. These conclusions may be confirmed with a direct cytometry measurement of anionic phospholipid exposure and comparison with another measure of $\alpha$-granule release, such as P-selectin expression.

The shear stress chosen for these studies is in the low range of what blood and platelets are exposed to in the vasculature. On the other hand, the $25 \%$ shear exposure time in the flow loop is higher than platelets are exposed to in a normal vascular circuit. Another possible complication is the shear-dependent interaction of platelets with vWf [3]. However, with regard to the latter, we note that gel-filtered platelets contain very little vWf compared with the plasma content, and our control experiments in the presence of anti-vWf antibody showed no reduction in platelet activation (fig. 2b).

That platelets activate under shear stress has long been known and is thought to be a major contributing factor to thrombotic disease, particularly in areas of high shear. These may be natural, as in the severe stenoses caused by arteriosclerosis, or artefactual, as is produced by the abnormal flow patterns produced by prosthetic heart valves. The results presented here strongly suggest that, at platelet counts $\geq 200,000 / \mu 1$, the bulk of the platelet prothrombinase activity generated under these experimental conditions is due to inter-platelet signaling rather than the direct result of shear stress, and that factor Va release is limiting. In contrast, the linearity of platelet activation that we observe at a count of 20,000 platelets/ $\mu$ indicates that platelet activation at such low counts is largely a direct result of shear stress exposure alone. (The fact that any prothrombinase activity is observed at all shows that some factor Va must be expressed even at low counts, but the amount is clearly sub-optimal.) This conclusion is relevant not only to the experimental measurement of shearinduced platelet activation in vitro, but also shows the importance of the platelet count in such studies. The pres- 
ent results suggest that for measurement of the direct effect of shear, platelet counts should be held as low as possible. This, we suggest, is true not only of pure studies of shear effects, e.g., in capillary loops or cone-plate viscometers, but also of studies of the platelet-activating potential of vascular devices like prosthetic heart valves.

\section{Acknowledgments}

This work was supported in part by a grant from the US National Science Foundation to D.B. (BES0302275). We also wish to thank our colleagues Wei Yin and Dave Rubenstein for their help and advice during this study.

\section{References}

-1 Brown CH 3rd, Leverett LB, Lewis CW, et al: Morphological, biochemical, and functional changes in human platelets subjected to shear stress. J Lab Clin Med 1975;86:462-471.

2 Hellums JD: 1993 Whitaker Lecture: biorheology in thrombosis research. Ann Biomed Eng 1994;22:445-455.

- 3 Kroll MH, Hellums JD, McIntire LV, et al: Platelets and shear stress. Blood 1996;88: 1525-1541.

-4 Wurzinger LJ, Blasberg P, Schmid-Schonbein $\mathrm{H}$ : Towards a concept of thrombosis in accelerated flow: rheology, fluid dynamics, and biochemistry. Biorheology 1985;22:437-450.

5 Bluestein D, Rambod E, Gharib M: Vortex shedding as a mechanism for free emboli formation in mechanical heart valves. J Biomech Eng 2000;122:125-134.

6 Yin W, Alemu Y, Affeld K, et al: Flow-induced platelet activation in bileaflet and monoleaflet mechanical heart valves. Ann Biomed Eng 2004;32:1058-1066.

7 Goto S, Handa S: Coronary thrombosis. Effects of blood flow on the mechanism of thrombus formation. Jpn Heart J 1998;39:579-596.

8 Peterson DM, Stathopoulos NA, Giorgio TD et al: Shear-induced platelet aggregation requires von Willebrand factor and platelet membrane glycoproteins Ib and IIb-IIIa. Blood 1987;69:625-628

-9 Moake JL, Turner NA, Stathopoulos NA, et al: Shear-induced platelet aggregation can be mediated by vWF released from platelets, as well as by exogenous large or unusually large vWF multimers, requires adenosine diphosphate, and is resistant to aspirin. Blood 1988;71: $1366-1374$
10 Kroll MH, Hellums JD, Guo Z, et al: Protein kinase $\mathrm{C}$ is activated in platelets subjected to pathological shear stress. J Biol Chem 1993 268:3520-3524.

11 Goto S, Salomon DR, Ikeda Y, et al: Characterization of the unique mechanism mediating the shear-dependent binding of soluble von Willebrand factor to platelets. J Biol Chem 1995;270:23352-23361.

12 Thiagarajan P, Tait JF: Collagen-induced exposure of anionic phospholipid in platelets and platelet-derived microparticles. J Biol Chem 1991;266:24302-24307.

13 Dachary-Prigent J, Freyssinet JM, Pasquet $\mathrm{JM}$, et al: Annexin $\mathrm{V}$ as a probe of aminophospholipid exposure and platelet membrane vesiculation: a flow cytometry study showing a role for free sulfhydryl groups. Blood 1993;81 2554-2565.

14 Ault KA, Rinder HM, Mitchell JG, et al: Correlated measurement of platelet release and aggregation in whole blood. Cytometry 1989;10 448-455.

15 Kane WH, Lindhout MJ, Jackson CM, et al: Factor Va-dependent binding of factor $\mathrm{Xa}$ to human platelets. J Biol Chem 1980;255:11701174.

16 Tracy PB, Giles AR, Mann KG, et al: Factor V (Quebec): a bleeding diathesis associated with a qualitative platelet Factor V deficiency. J Clin Invest 1984;74:1221-1228.

17 Tracy PB, Eide LL, Mann KG: Human prothrombinase complex assembly and function on isolated peripheral blood cell populations. J Biol Chem 1985;260:2119-2124.

18 Gould WR, Silveira JR, Tracy PB: Unique in vivo modifications of coagulation factor $\mathrm{V}$ produce a physically and functionally distinct platelet-derived cofactor: characterization of purified platelet-derived factor V/Va. J Biol Chem 2004;279:2383-2393.
19 Rubenstein D, Jesty J, Bluestein D: Differences between mainstream and sidestream cigarette smoke extracts and nicotine in the activation of platelets under static and flow conditions. Circulation 2004; 109:78-83.

20 Hellums JD, Peterson DM, Stathopoulos NA et al: Studies of the mechanism of shear-induced platelet activation; in Hartman A, Kushinsky W (eds): Cerebral Ischemia and Hemorheology. New York, Springer, 1987, p 80.

21 Jesty J, Yin W, Perrotta P, et al: Platelet activation in a circulating flow loop: combined effects of shear stress and exposure time. Platelets 2003; 14:143-149.

22 Jesty J, Bluestein D: Acetylated prothrombin as a substrate in the measurement of the procoagulant activity of platelets: elimination of the feedback activation of platelets by thrombin. Analyt Biochem 1999;272:64-70.

23 Martin DW, Jesty J: Calcium stimulation of procoagulant activity in human erythrocytes. ATP dependence and the effects of modifiers of stimulation and recovery. J Biol Chem 1995; 270:10468-10474.

24 Beltrami E, Jesty J: Mathematical analysis of activation thresholds in enzyme-catalyzed positive feedbacks: application to the feedbacks of blood coagulation. Proc Natl Acad Sci USA 1995;92:8744-8748.

25 Sims PJ, Wiedmer T: Unraveling the mysteries of phospholipid scrambling. Thromb Haemost 2001;86:266-275.

26 Bahou WF, Scudder L, Rubenstein D, et al: A shear-restricted pathway of platelet procoagulant activity is regulated by IQGAP1. J Biol Chem 2004;279:22571-22577. 